\title{
Fractal and Image Analysis of Collagen Fibrosis in Hypertensive Induced Myocardial Remodeling in Mouse Hearts Lacking STAT3 Protection
}

\author{
John W. Fuseler ${ }^{1}$, Fouad A. Zouein ${ }^{2}$, Mazen Kurdi ${ }^{2}$ and George W. Booz ${ }^{2}$
}

1. University of South Carolina, School of Medicine, Columbia South Carolina. 29209

2. University of Mississippi Medical Center, School of Medicine, Jackson, MS 39216-4505

With hypertension, the left ventricle of the heart undergoes hypertrophy, which initially is beneficial for normalizing wall stress and maintaining cardiac function. However, cardiac hypertrophy and the associated remodeling of the extracellular matrix resulting from sustained hypertension are detrimental eventually leading to heart failure. How the myocardium initially adapts to increased wall stress is poorly understood, as are events responsible for transitioning to heart failure. Loss or reduction in the initial adaptive response may contribute to the progress to failure. The transcription factor, STAT3, is involved in protection of the heart by ischemic preconditioning. However, the role of STAT3 in the heart in chronic stress is unclear. We previously showed that STAT3 is important in hypertensioninduced cardiac remodeling using mice with reduced STAT3 activity due to a S727A mutation (SA/SA). Wild type (WT) and SA/SA mice received angiotensin (ANG-II) to induce the hypertensive conditions and were compared with control mice treated with saline [1]. Loss of STAT3 was associated with development of cardiac dysfunction marked by a combined loss of myofibrillar content of cardiac myocytes and a reduction in the mass of contractile filaments. Additionally, attenuated STAT3 activity resulted in alterations in cardiac remodeling as evidenced by the presence of unique patterns of collagen fibrosis deposition. In this study, we applied image analysis and determination of the fractal dimension (D) to quantify these patterns of fibrosis in the myocardium in response to reduction in STAT3 expression and induction of cardiac hypertension by ANG-II.

Materials and Methods: Fixed hearts were cut laterally through the mid region to cross sections of both the left and right ventricles, embedded in paraffin, sectioned ( $5 \mu \mathrm{m}$ thickness), and stained with Masson's Trichrome stain for collagen morphology. Sequential, adjacent images (15-20 images per section) were collected as TIFF files and morphometric analysis carried out using MetaMorph 6.1 and HarFA analytical software. The fibrotic regions in the experimental hearts and the collagen matrix in control heats were isolated as ROIs and morphological descriptors of area (A), integrated optical density (IOD), $\%$ fibrotic collagen and the fractal dimension (D) measured. The upper and lower threshold range values defining the collagen matrix in the fibrotic regions in the SA/SA+ANG-II hearts were recorded and applied to all groups to evaluate equivalent fibrotic lesions. In the control and experimental hearts, the collagen matrix was analyzed and compared by measurements of fibrotic collagen (\% collagen), its chaos (D value) and relative density (IOD/A). A minimum of 20 images were analyzed from each heart section of each animal in the controls and experimental groups. Three to five mice were analyzed for each group.

Results: The morphological descriptor of collagen fibrosis resulting for the induction of hypertension and loss of STAT3 protection are summarized in Table 1. 
Table 1. Summary of the morphological descriptors of fibrotic collagen

\begin{tabular}{|l|l|l|l|}
\hline Treatment Group & \% Area Fibrotic Collagen & IOD/A (Relative Density) & D \\
\hline WT+Saline & $6.56 \pm 0.82$ & $1.682 \pm 0.077$ & $1.434 \pm 0.013$ \\
\hline WT+ANG-II & $7.18 \pm 0.74$ & $2.211 \pm 0.160$ & $1.446 \pm 0.015$ \\
\hline SA/SA+ Saline & $13.30 \pm 1.62$ & $3.200 \pm 0.151$ & $1.528 \pm 0.019$ \\
\hline SA/SA+ANG-II & $35.90 \pm 2.99$ & $2.715 \pm 0.067$ & $1.753 \pm 0.013$ \\
\hline
\end{tabular}

1. Effect of Hypertension on collagen remodeling: Induction of hypertension in the WT mice by ANG-II resulted in a non-significant increase in the \% Area and relative density of the fibrotic collagen. The D values in the presence of ANG-II remained constant. These changes appear to be sufficient induce changes in hemodynamic parameters observed in hypertension. The increase in collagen matrix density without an increase in chaos of the structure may serve to strengthen the myocardium in response to hypertension.

2. Loss of STAT3 protection induces significant alteration in the collagen extra cellular matrix: Suppression of STAT3 resulted in extensive increase in the amount and remodeling of myocardial collagen characterized by the induction of focal regions of fibrosis and loss of myocytes. The SA/SA+ saline hearts exhibited significant increases in fibrotic collagen $(\mathrm{P}=0.025)$ and density $(\mathrm{P}<0.001)$ compared to the controls. The $\mathrm{D}$ values indicate a significant $(\mathrm{P}=0.002)$ increase in chaos associated with the collagen matrix and developing fibrotic foci.

3. Induction of hypertension in hearts lacking STAT3 protection results in extensive remodeling of the collagen extra cellular matrix: Treatment of the SA/SA mice with ANG-II resulted in dramatic and extensive remodeling of collagen in the myocardium associated with loss of myocytes within the fibrotic regions. These fibrotic regions were larger with the appearance of a meshwork array of collagen radiating from a central region. The presence of ANG-II in the SA/SA myocardium induced further significant increases $(\mathrm{P}<0.001)$ in the amount of collagen, the relative density of the collagen in the $\mathrm{SA} / \mathrm{SA}+\mathrm{ANG}-\mathrm{II}$ group was significantly less $(\mathrm{P}=0.015)$ less than the $\mathrm{SA} / \mathrm{SA}+$ saline group but still significantly greater than the WT+saline and WT+ANG-II groups. This decrease in collagen density was associated with a large significant $(\mathrm{P}<0.001)$ increase in its $\mathrm{D}$ value. This indicates the collagen associated with these fibrotic foci is high disorganized and more space filling as it fills the voids created by the loss of myocytes. Progressive loss of myocytes and their replacement by chaotically and randomly organized collagen would serve to further weaken the myocardium leading to cardiac failure.

Characterization of fibrotic foci by quantitative parameters, especially the $\mathrm{D}$ values, could be beneficial in identification and diagnosis of different types of fibrosis associated with cardiac pathologies, when correlated with associated changes in hemodynamic functions, electrical conduction and altered biochemical expression. Additionally, STAT3 expression appears to be essential in normal regulation and organization of the collagen matrix in the myocardium associated with normal cardiac function. Suppression of STAT3 results in fibrotic lesions in the myocardium which are exacerbated by hypertension.

Reference.

[1]. Zouein FA, Zgheib C, Hamza S, Fuseler JW, Hall JE, Soljancic A, Lopez-Ruiz A, Kurdi M, Booz GW. Hypertens Res. 2013 Jan 31. doi: 10.1038/hr.2012.223. [Epub ahead of print]. 